\title{
MODE OF ACTION OF CHEMOTHERAPEUTIC AGENTS
}

$\mathrm{T}$ HE Biochemical Society held a discussion meeting on the mode of action of chemotherapeutic agents on November 29 at the Courtauld Institute of Biochemistry, Middlesex Hospital, Prof. E. C. Dodds being in the chair.

Dr. G. M. Findlay, in opening the discussion, said that chemotherapeutic action can be classified as direct or indirect. Except in the case of parasites present in the intestinal canal, it is essential that the chemotherapeutic drug should be absorbed into the body, that it should penetrate to the site where the parasites are acting, and that it should not be excreted or converted too rapidly into an inert form. Time must be allowed for chemotherapeutic action, and in some cases for the conversion of the compound from an inactive into an active form.

When once the drug and the parasite have been brought face to face, three stages can be distinguished, adsorption, interference with metabolism, and death or such injury to the parasite that it is destroyed by the phagocytes of the host. An absorbed chemotherapeutic drug may prevent an essential food factor from being adsorbed or it may cause a breakdown in metabolism by combining with a specific substrate or by competing with an essential cell metabolite for an enzyme or coenzyme. One break in the chain of metabolic reactions may rapidly give rise to others. Specific immune serum and sulphapyridine do not compete for the same receptor group in the pneumococcus and may therefore enhance one another.

Parasites may be killed in the body without the aid of phagocytes, but usually when a parasite has been damaged it is destroyed by the normal defence mechanism of the host.

Indirect action produces such changes in the environment that parasites can no longer grow. Physical changes may prevent growth, the temperature or the $p \mathrm{H}$ reaction may be altered, the formation of immune bodies may be stimulated or the character of the cells may be altered, as in the treatment of gonococcal vulvovaginitis in children with œstrin preparations. The highly specific action of certain drugs and the no less specific reactions of certain closely allied parasites can be explained by postulating that, after adsorption of the compound at the parasite/solution interface, the nature of the interference with the metabolism of the parasite depends on what groupings in the molecule of the compound come within the influence of other acceptor groups in the parasite: there is thus a multipoint action.

Prof. A. Fleming, continuing the discussion, said that in 1929 he applied the name penicillin to an anti-bacterial substance of unknown constitution elaborated by Penicillium notatum when grown in ordinary bacteriological media or in a modified Czapek-Dox medium. The action is mainly bacteriostatic and shows a marked degree of specificity. Pyoganic cocci, Clostridia and some other bacteria are sensitive, while the coli-typhoid, hæmophilic chromogenic bacilli and others are insensitive. Pathogenic Gram-negative cocei (Gonococcus, Meningococcus and $M$. catarrhalis) are sensitive, while saprophytic varieties, for example $M$. flavus, are insensitive, thus differing from the sulphonamides.

The action of penicillin is not interfered with by substances that inhibit sulphonamides, bacteria, bacterial extracts, pus fluids, tissue autolysates, peptones and $p$-aminobenzoic acid. Penicillin is non-toxic to leucocytes and animals, but in low dilutions it affects the morphology of bacteria and interferes with division. As penicillin and also gramicidin are apparently of a different constitution from the sulphonamides, the isolation and synthesis of the pure active principles will open up a new chemotherapeutic field.

Prof. Warrington Yorke described the action of the aromatic diamidines which were evolved by Dr. Ewins, after it had been shown that synthalin (decane diguanidine) acts on trypanosomes not by reducing the sugar but by direct toxic action. Some aromatic diamidines exhibit a remarkable trypanocidal activity. The most active are $4: 4^{\prime}$-diamidinostilbene, $4: 4^{\prime}$-diamidino-diphenoxy-propane and 4 : $4^{\prime}$-diamidino-diphenoxy-pentane. Cases of kala-azar have now been successfully treated by diamidinestilbene-Indians, children in the Mediterranean area and patients with the Sudanese variety, which is resistant to antimony. Babesia infections in dogs have been cured by the stilbene and propane derivatives, the stilbene being highly active against Trypanosoma congolense. The compounds have a highly specific action. Drug resistance appears to be due to a change in the surface layer of the parasite. Trypanosomes resistant to a number of compounds have now been prepared, including a diamidine preparation. It has also been possible to prepare a diamidine-resistant Babesia and a plasmoquineresistant strain of Plasmodium knowlesi in the monkey.

Discussing trypanocidal substances, Dr. F. Hawking said that from the phenomenon of drug resistance four kinds of receptors on the trypanosome are recognized. Trypanocidal action comprises fixation of the drug, səcondary chemical reactions inside the cell and death. Only about the first process is much known : it occurs quickly in a few minutes and is reversible. With arsenical compounds, fixation apparently depends on the tervalent arsenic atom linked to a benzene ring. Certain side chains $\left(-\mathrm{N}^{\prime} \mathrm{H}_{2},-\mathrm{OH}\right)$ prevent fixation on animal cells but not on normal trypanosomes: the receptors of resistant trypanosomes are modified. Over a wide range, the amount of drug fixed is proportional to the concentration in the surrounding fluid: in the case of acriflavine the partition ratio (concentration inside the trypanosome/concentration outsid $\rightarrow$ ) is 8,000 for normal trypanosomes, 60 for resistant trypanosomes. Fluorescent compounds, acriflavine and diamidino stilbene, are concentrated in the blepharoplast and cytoplasmic granules of the trypanosome.

The importance of having a theory of chemotherapeutic action was emphasized by Sir Henry Dale, who congratulated the Society on arranging a discussion meeting in war-time. It was one of Paul Ehrlich's great contributions to the subject to have produced theories which, though they will probably not survive unmodified, have given tremendous stimulus to research; thus, his explanation of the action of certain dyes on infection by trypanosomes as due to injury of the reproductive power of the trypanosomes, without affecting their other vital functions. 
At the time, this suggestion seemed artificial and unconvincing, but when Dobell and Laidlaw found a method for growing Entamceba histolytica in per. manent eulture in vitro, it could be demonstrated that the action of emetine was just of this type. The 'factor of persistence' is also of importance in chemotherapeutic activity. This seems to be the reason why a quinquevalent arsenical on one hand, or an arseno-compound on the other, is a better chemotherapeutic agent than the arsenoxide produced by reduction from one or by oxidation from the other, although the arsenoxide is recognized as the directly parasiticidal agent. In the same way, sulphaguanidine seems to owe its effectiveness in bacterial dysentery to its poor solubility, enabling it to remain in solid form in the intestinal contents and to keep up a steady, low concentration in contact with the infected mucous membrane. Aromatic diamidines, the brilliant promise of which has been made clear by the results reported by Prof. Warrington Yorke, may similarly owe part of their superiority to their limited solubility. Sir Henry suggested, as problems the solution of which might greatly accelerate advance in parts of the field of chemotherapy, the discovery of a method of keeping trypanosomes alive and reproductive indefinitely in artificial culture, and the discovery of a method of treating a strain of trypanosomes which has acquired a drug resistance, so as to restore the normal susceptibility.

Dr. D. D. Woods spoke of the interference of antibacterial agents and essential metabolites. This interference may be due to the formation of a compound between the antibacterial agent and the essential metabolite, as between mercury salts and - SH compounds, or by inhibition of an enzyme reaction involved in the synthesis or utilization of an essential metabolite. An example of this is the competitive inhibition by sulphanilamide of an enzyme reaction involved in the further utilization of $p$-aminobenzoic acid, this inhibition occurring by virtue of the chemical relationship of sulphanilamide and $p$-aminobenzoic acid. The latter has been isolated from natural sources and is a growth factor for Clostridium acetobutylicum and higher organisms. Following out this hypothesis, the following substances all chemically related to a known essential metabolite, the latter shown in brackets, have been found to have some antibacterial activity ; pyridine3-sulphonic acid and amide (nicotinic acid and amide); aminosulphonic acids (analogous aminocarboxylic acid); sulphonic acid analogue of pantothenic acid (pantothenic acid); indole-3-acrylic acid (tryptophan); barbituric acid (uracil).

Dr. H. McIlwain said that an organism which is deprived of the use of enzymes or metabolites by various types of interference is nutritionally more exacting than in its normal state. Bacterium coli and Streptocnccus homolyticus, inhibited by acriflavine components, require for further growth two types of material not normally required. Type 1 is best replaced by nucleotides, type 2 by a concentrate of amino acids but partly by phenylalanine. In the presence of type 2 compounds, but not without, artificial hydrogen carriers are further active against inhibition of Bact. coli. Type 1 compounds form complex salts with acriflavine components. The inhibitors probably inactivate enzyme systems of which type 1 compounds are essential parts, of which type 2 compounds are substrates or products and of which some can be replaced by the hydrogen carriers.
The relationship between chemical constitution and bactericidal action in certain amino-acridines was described by Dr. W. H. Linnell. Among the isomeric diaminoacridines, a'1-amino group causes complete loss of bactericidal activity and reduces toxicity; a 2-amino group increases activity and this is further enhanced by another amino group in the 2-, 3-, 4-, (in the other ring) or 5-positions, accompanied by increased toxicity in the case of a second 2-substituent. When two 3-amino groups $(=3: 7)$ are present, activity is moderate but the $3: 8$ diamino acridine $(=2: 7)$ is as active as proflavine but less toxic. A 4-amino substitutent confers small activity, while position 5 is highly active, but probably leads to increased toxicity. Albert has shown that similar differences in activity exist among the five isomeric-amino acridines, their activity paralleling their strength as bases and their partition coefficients between oil and water. The corresponding acridones are inactive, as are certain amino derivatives of 5 : 10-dihydroacridine and of iminodihydroacridine, suggesting that the intact acridine molecule is necessary.

L. G. Goodwin said that the uncertain action of antimony in protozoal diseases, of which the resistance of Sudanese kala-azar to antimony is an example, is an added difficulty in investigating its mode of action. The active form of antimony may be the stibinoxide grouping, but while this is probable in trypanosome and schistosome infections, it is unlikely in leishmaniasis, where quinquevalent compounds are the most effective and massive dose therapy is successful.

Excretion of antimony after doses of the quinquevalent compounds or of stibophen is much more rapid than with tartar emetic. There is indirect evidence that the rapidly excreted fraction of the drug passes through the body unchanged.

Both direct toxicity action on parasites and stimulation of the hosts' defence mechanisms are produced by antimonials. Increased phagocytosis may be of primary importance in leishmaniasis, though histological work on the spleens of infected hamsters injected with a quinquevalent antimony compound suggest some degree of direct action.

Dr. E. Chain described the chemical and physical properties of penicillin in relation to its bacteriostatic action. It is a strong acid with two, or a multiple of two, acid groups. A purified barium salt gives a carbon content of 55 per cent and a hydrogen content of 6.3 per cent : only carbon, hydrogen and oxygen are -present in the molecule. Methoxyl groups cannot be detected but two hydroxyl groups are present. The dried barium salt of penicillin keeps indefinitely and in watery solution is most stable between $p H$ and 7. With heavy metals, except $\mathrm{Fe} \cdots$, it forms water-soluble salts. The antibacterial action is lost by oxidation with hydrogen peroxide and potassium permanganate. Dr. E. P. Abraham said that the instability of penicillin necessitates three methods of purification dependent on distribution between solvents, adsorption and reduction. The crude barium salt obtained from an amyl acetate extract of the medium has an activity of $15-25$ units per mgm. Distribution between water and ether at $p \mathrm{H} 2$ and $p \mathrm{H}$, adsorption of impurities by charcoal and chromatographic analysis on alumina yield a light yellow barium salt with an activity of about 150 units per mgm. On reduction of this material in neutral solution with aluminium-mercury couple the remaining pigment is adsorbed by alumina. The 
white barium salt obtained from the supernatant has an activity of 240 units per mgm. It completely inhibits Staphylococcus in a dilution of 1 in 5,000,000, partially in a dilution of 1 in 16,000,000.

The behaviour of sulphanilamide, $p$-aminobenzoic acid and chemically related compounds, aniline and sodium benzenesulphonate, at the surface of Bact. coli has been studied by Dr. F. R. Bradbury and D. O. Jordan by electrokinetic methods. The shapes of the curves relating variation of mobility with time of contact for sulphanilamide and $p$-aminobenzoic acid are quite different from those of the curves for aniline and sodium benzenesulphonate. The curves for sulphanilamide and $p$-aminobenzoic acid are similar, suggesting that the two compounds behave in a like manner at the bacterial surface.

Prof. A. St. G. Huggett said that dyes such as chlorazol sky blue F $P S$ (Chicago blue) and chlorazol fast pink $B K S$ are excellent anticoagulants. Structurally, they resemble afridol violet, from which Bayer 205 is derived; they have a trypanocidal action while Bayer 205 has an anticoagulant action. The dyes act at two points in the clotting mechanism as antikinase and antithrombin. Their anti-enzyme action with blood clotting may have an analogy to their mechanism as trypanocidal agents.

\section{CO-ORDINATION OF SCIENTIFIC AND TECHNICAL WORK}

\begin{abstract}
A a conference organized by the Association of A Scientific Workers and held at Birmingham on December 6, the need for greater co-ordination and collaboration in all fields of applied science was urged. The essential part which the scientific worker has to play in the modern community was emphasized by Mr. D. P. Riley, who opened a discussion on "The Responsibilities of the Scientist to the Community". Not only most of the greatest industries but even agriculture are dependent upon his work if they are to develop rapidly enough to satisfy the needs of the whole community, and in time of war it is even more important that this should be recognized, particularly in view of the five years' start which our enemies have over us in the application of science to war problems. As an interesting example of the need for the proper understanding of the scientific facts underlying certain decisions by those entrusted with executive power, Mŕ. Riley took the case of the encouragement of the consumption of wholemeal bread on account of its vitamin B content. The fact that this bread also contains an appreciable amount of phytic acid, the calcium salt of which is insoluble, and the consequent danger of avoiding vitamin B deficiency only at the expense of incurring calcium deficiency, has apparently not yet been given attention in public policy. Not only are many scientific workers still not occupied to their full capacity, but they are even in some cases urged to give their attention to the development of post-war plans, as well illustrated by an advertisement in a well-known daily paper describing a sewing-machine made largely of plasties and saying that now is the time to plan its post-war production.
\end{abstract}

The Association of Scientific Workers, which is working to secure the fullest application of science in the service of the community and a responsible status for men of science, is trying to meet the need for organization without which the individual man of science can do little in his attempt to ensure that his work is used for the war effort. In particular, the Association wishes for any evidence of definite misapplication or inefficiency in the use of scientific man-power, since it has been requested by the Ministry of Supply to prepare a report on this subject.

The immediate problems of producing and maintaining equipment for our armed forces was dealt with by Mr. Swann in his address on "The Role of the Scientist in the National Effort". He produced figures to demonstrate the superiority of Germany and the occupied countries over ourselves and the U.S.S.R., including also the help given by the United States, leaving no ground for optimism. He men. tioned the dissipation of effort in competition between private firms, the operation of the 'cost-plus' contract, inadequate pooling of information and the determined grip of firms on their trade secrets as some of the many factors combining to hold up production, and criticized the feeling of complacency fostered by Russia's successes and America's promises of support. $\mathrm{He}$ urged the formation of production committees, where these do not already exist, on which technical staff should play an active part, and the need to provide the Ministry of Supply with all relevant facts as to means to eradicate inefficiencies.

Mr. J. A. Henley pointed out how, as the industrial scientist has passed from the position of being an independent consultant or even his own manager to that of one wage-earner among many in a large firm, it has become necessary for him to co-operate with his fellows in a professional organization which could look after his interests and his status. This need has become peculiarly great since the outbreak of war ; hence it is not surprising that the Association of Scientific Workers has grown very much faster since its registration as a trade union than formerly.

After discussion, the following resolutions were passed :

"The Birmingham Conference of Scientific and Technical Workers reciprocates the expressed desire of the scientists of the U.S.S.R. for the fullest possible co-operation in the fight against Fascism, and pledges its maximum efforts to this end.

"This Conference, realising that maximum efficiency in the war effort will be achieved only as a result of fullest possible co-operation between technical staffs, work-people, and those in control of production, supports all activities leading to this end, in particular the setting up of production committees.

"This Conference calls for complete pooling of technical information between manufacturing companies with similar problems.

"This Conference stresses that the present anomalies in conditions of working and remuneration must lead to grievances that seriously hamper the war effort." 